\title{
DVB-T2: An Outline of HDTV and UHDTV Programmes Broadcasting
}

\author{
Milan Milivojević, Božimir Mišković, and Irini Reljin, Senior Member, IEEE
}

\begin{abstract}
Increasing of video resolution of the HD programmes requires a higher bit rate. On the other hand, a continuous enhancement of existing and introduction of new source coding techniques are evident. Technical evolution of the Digital Terrestrial Television platform improves channel coding and RF channel utilization. Some optional solutions have also been proposed. This paper discusses the possibilities of allocating HD programmes in formats expected in the near future. Performed calculations are guidance to accurate examination of the efficiency of allocating high definition programmes in the DVB-T2 multiplex, in line with existing and expected enhancements.

Keywords - High definition television; ultra high definition television; high efficiency video coding; terrestrial digital video broadcasting; utilization; broadband statistical multiplexing; time frequency slicing; channel efficiency; statistical gain; network planning gain.
\end{abstract}

\section{INTRODUCTION}

$\mathrm{I}_{\mathrm{ex}}^{\mathrm{N}}$ terms of television signal quality, consumers' expectation as well as TV set capabilities, there are dramatical changes. There is a trend for displays that requires higher resolutions and frame rates, dimensions from 42 inches and larger, and most are already adapted to HD (High Definition) format 1080p. Broadcasting of the first generation of HD formats $1080 \mathrm{i}$ and $720 \mathrm{p}$ has already begun, while HD 1080p format is expected. Better video quality, in terms of increasing other performances, such as aspect ratio, chrominance resolution, bit depth and colour space has already been disscussed. The broadcast of the 3DTV video has slightly increased, and it largely depends on the development of compression techniques. Currently, efforts are directed towards the specifications for broadcasting 3DTV videos with multiple views.

Paper received July 6, 2015; accepted July 19, 2015. Date of publication November 15, 2015. The associate editor coordinating the review of this manuscript and approving it for publication was Prof. Dušan Drajić.

This paper is a revised and expanded version of the paper presented at the 22th Telecommunications Forum TELFOR 2014.

This research is supported and partially funded by the Ministry of Education, Science and Technological Development of the Republic of Serbia, under the project TR32048.

Milan Milivojević is with the School of Electrical Engineering University of Belgrade, Bulevar kralja Aleksandra 73, 11120 Belgrade, Serbia (phone: 381-61-1312602; e-mail: msmilance@etf.rs).

Božimir Mišković is with Telekom Srbija, Gospodar Jovanova 15 , 32000 Čačak, Serbia (phone: 381-32-221133, e-mail: bozimir@telekom.rs).

Irini Reljin is with the School of Electrical Engineering, University of Belgrade, Bulevar kralja Aleksandra 73, 11120 Belgrade, Belgrade, Serbia (e-mail: irinitms@gmail.com).
Improvement of channel coding, and the ability to view video without special glasses has already been achieved. Commercial use of UHDTV (Ultra High Definition Television) and SHDTV (Super High Definition Television) formats with $4 \mathrm{~K}(2160 \mathrm{p})$ and $8 \mathrm{~K}(4320 \mathrm{p})$ resolutions $[1,2]$ is announced for the near future .

It is expected that laptops, tablets and mobile phone devices will have high quality reception of video content. Since the screen size of these devices is increased and the content is viewed from much shorter/smaller distances, the required resolution to achieve the same quality must be even higher than in stationary devices used in home conditions.

A common factor of all new programme formats and improvements is to increase the required bit rate. It consequently reduces the number of programmes allocated in multiplex of RF channels. On the other hand, customers expect expansion of TV programmes. The task is clear and the solutions can be sought in several directions:

- reduction of bit rate per programme by increasing the efficiency of source coding,

- increasing the capacity of RF channel,

- improving utilization of this capacity, and

- increasing the percentage of payload bits in the total bit rate.

The source coding equipment plays a crucial role in the reduction of original data rate while the quality degradation is acceptable. Every ten years, there is a new technique for coding the source stream. In this period of time, the level of compression plays a very important role, since, in addition to the increased data rate per programme, the number of offered TV programmes grows as well.

This paper observes data rate values for the current HD formats (720p, 1080i and 1080p) encoded with H.264/AVC protocol [3], [4], and assumes that expected data rates for current and future HD formats (1080p, 3DTV based on 1080p and 2160p) are encoded according to new protocol H.265 [5], [6]. Initial tests indicate that this protocol, also known as HEVC (High Efficiency Video Coding), provides an improvement by a factor of 2 over H.264 regarding coding efficiency. During the period of exploitation, according to experience with previous coding techniques H.262 (MPEG-2) and H.264/AVC (MPEG-4), a further increase in efficiency of H.265 is expected.

The second generation of DTT platform (Digital Terrestrial Television) called DVB-T2 (Digital Video Broadcasting - Terrestrial) [7], [8] adopted in Europe, increases the data rate of multiplex within a single RF 
channel roughly by $50 \%$, compared to the first generation (DVB-T). This is more than the required capacity for SD (Standard Definition) programmes. However, these new resources can accommodate a relatively small number of the first generation of HD programmes in $720 \mathrm{p}$ and $1080 \mathrm{i}$ formats. Only 4 to $6 \mathrm{HD}$ programmes per multiplex can be allocated, while the bandwidth efficiency is insufficient.

Besides the high data rates $(6$ to $10 \mathrm{Mb} / \mathrm{s}$ per programme), an additional shortage is the VBR (Variable Bit Rate) nature of compressed video content. For example, data rate variation in HD 1080p is between 6 and $14 \mathrm{Mb} / \mathrm{s}$ [9]. In CBR (Constant Bit Rate) each programme is allocated with appropriate constant bit rate capacity. In order to preserve the quality and taking into account the upper limit of the rate, a vast capacity is required. It is clear that in intervals with a low bit rate, capacity will be used inefficiently.

The problem can be solved using statistical multiplexing [7], [10]. This technique is proposed for common compression and multiplexing of all programmes assigned to multiplex. Here the efficiency increases with the number of programmes. It does not accomplish the full effect with the HD programmes. Actually, if the rate per programme is not reduced sufficiently, for example by applying HEVC, the capacity of multiplex will not be completely utilized.

Estimations of expected data rates for different services with applied H.265 technique in this research are used from [11], [12]. They give a realistic insight into the possible number of programmes in various formats within the multiplex planned under DVB-T2. In order to improve broadcasting efficiency, primarily 3DTV and UHDTV services, all analyses have been performed using proposed implementation of the Time Frequency Slicing (TFS) techniques, optionally provided in DVB-T2 $[7,13]$. The results suggest compression ratios and modalities of multiplexing techniques required for the efficient broadcast in $4 \mathrm{~K}$ resolution.

\section{Allocation of HD PROGRAmmes}

State of the art of HD programmes in HD720p and HD1080i formats, with H.264/AVC coding technique, assumes the capacity of about $7 \mathrm{Mb} / \mathrm{s}$ reserved within multiplex. Constant improvements of H.264 codecs promise the reduction of data rate in the future. In the future, programmes in HD720p, and HD1080p formats are expected. In this paper we assume programmes within the multiplex with the CBRs of $7.5 \mathrm{Mb} / \mathrm{s}$ and $10 \mathrm{Mb} / \mathrm{s}$ for one programme. For 3DTV formats, based on HD1080p, a bit rate of $15 \mathrm{Mb} / \mathrm{s}$ is considered. Due to VBR nature of compressed TV bit stream, proposed content has a roughly $30 \%$ greater capacity than the average television programme data rates. Nevertheless, it is quite possible that, at some instant, this capacity becomes insufficient and it further results in loss of data and quality degradation.

Statistical multiplexing provides full RF channel capacity to all programmes within the multiplex. Adjustments of multiplexer and VBR compression encoder resources lead to more efficient usage of multiplex capacity and increase of allocated programmes. It is assumed that the data rate variations of programmes are uncorrelated. If the number of programmes increases, statistical equilibrium is more likely attainable. The total bit rate of multiplex is almost constant, while the rates of certain programmes are variable.

The goal is to provide a sufficiently large number of multiplexed programmes within the multiplex. Thus the capacity of multiplex sholud be as close as possible to the sum of average data rates of individual programmes. As it is shown in Fig. 1, taken from [10], as the number of channels increases, programmes from the multiplex can be allocated with a $30 \%$ lower data rate capacity compared to a single CBR allocation where the number of programmes is more than 20. For example, statistical multiplex with 10 programmes provides a $25 \%$ lower bit rate with the same quality of service. This means that each programme uses on average $75 \%$ of the data rate that is reserved for the same programme in CBR allocation mode.

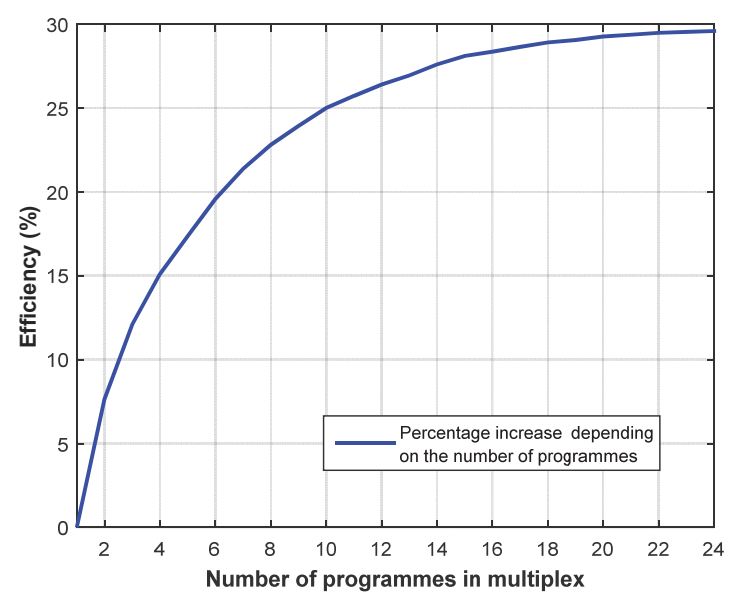

Fig. 1. Statistical multiplexing efficiency depending on the number of programmes.

However, multiplex of HD programmes in a single RF channel, in e.g. MFN (Multi Frequency Network) transmission mode, supports 4 to 6 programmes, that is much less than 15 to 18 programmes in SD (Standard Definition) format. Statistical multiplexing increases the utilization of HD programmes from 15 to $18 \%$. That is a significant improvement, but still lower than the $28 \%$ achieved with SD programmes.

Statistical multiplexing has several shortcomings. It is a complex and demanding process that, first of all, requires demultiplexing of each compressed programme individually. Statistical multiplexer at the transmitter and the receiver side should have a great processing power and memory resources and the whole processing procedure must be done in real time. Errors can occur in programmes with scrambled video content, since the multiplexer must be provided with the applied logic protection.

Fig. 2. shows capacities required for the programmes of certain formats with and without statistical multiplexing. For future UHDTV programmes in $4 \mathrm{~K}$ format, the bit rate estimation is only illustrative, because it is not planned for UHDTV programmes to be encoded with H.264 protocol. Horizontal line represents capacity of a single multiplex 
for a fixed MFN network. Capacity per multiplex in a single SFN and portable/mobile DVB -T2 networks is even smaller and it is not shown in order to avoid confusion.

It is obvious that the effect of statistical multiplexing is relatively small since the number of programmes per multiplex does not exceed 7 even in the least demanding HD720p format. Shaded segments in Fig. 2 show the effect of statistical multiplexing of the programme (upper line), compared to the CBR capacity allocation (lower line).

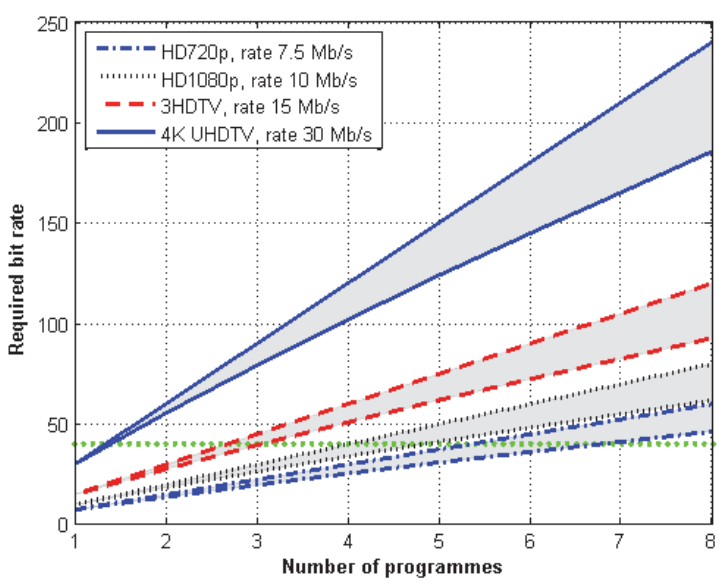

Fig. 2. Number of programmes per channel without and with statistical multiplexing (H.264/AVC).

Improvement obtained by statistical multiplexing is represented by very narrow segments due to the small number of programmes per multiplex and it shows that the efficiency is increased insignificantly. Thus, the application of statistical multiplexing of HD programmes in a single RF channel does not provide efficient capacity utilization. The bit rate variations within the multiplex are large and it reflects on fluctuations of content quality. Placing only one $4 \mathrm{~K}$ format programme into a single channel multiplex is not a reasonable solution.

Obviously, implementation of H.264/AVC compression technology in a single RF channel is reserved only for SD programmes. In addition, the first generation of $\mathrm{HD}$ programmes (HD720p and HD1080) significantly reduces the efficiency when the number of programmes is increased.

\section{EXPECTATION OF H.265}

It is clear that HD programme multiplexing becomes much more efficient when H.265 codec is implemented. Increase of the number of programmes influenced by efficient compression is followed by the increase of the number of programmes, influenced by expanded efficiency of statistical multiplexing in accordance with Fig. 1. The question is how compression should be improved in order to obtain efficient utilization of the channel capacity.

Analyses with the objective methods have shown that the same quality is possible to reach with a reduced bitrate of around $40 \%$ compared to the H.264/AVC [11]. The subjective analyses show that the bit rate can be reduced by more than $60 \%$ [12]. It is a rational assumption that in the initial commercial use, compression will be doubled (rate reduction coefficient $\mathrm{k}=0.5$ ).

The expected bit rates for different HD formats are given in Table1, where the source stream is encoded with H.264/AVC or H.265 coding technique. H.265 compression is discussed for two scenarios: when the compressed stream is reduced to $50 \%$, or even to $40 \%$ of the rate compressed with H.264/AVC protocol (rate reduction coefficient $\mathrm{k}=0.4$ ). The number of statistically multiplexed HD programmes, will be accordingly increased more than twice, because of the better multiplex efficiency.

TABLE 1: ASSUMED BIT RATE FOR PROGRAMME ENCODED BY H.264/AVC FOR DIFFERENT FORMATS AND TWO VARIANTS OF H.265.

\begin{tabular}{c|c|c|c|c}
\hline Rate $(\mathbf{M b} / \mathbf{s})$ & $\begin{array}{c}\boldsymbol{H D} \\
\mathbf{7 2 0 p}\end{array}$ & $\begin{array}{c}\boldsymbol{H D} \\
\mathbf{1 0 8 0} \boldsymbol{p}\end{array}$ & $3 \mathbf{3 D V}$ & $\begin{array}{c}\boldsymbol{H D} \\
\mathbf{2 1 6 0 \boldsymbol { p }}\end{array}$ \\
\hline H.264 & 7.5 & 10.00 & 15.00 & 25.00 \\
H.265 & 3.75 & 5.00 & 7.50 & 12.50 \\
$\mathrm{k}=0.5$ & & & & \\
$\begin{array}{l}\text { H.265 } \\
\text { k=0.4 }\end{array}$ & 3.00 & 4.00 & 6.00 & 10.00 \\
\hline
\end{tabular}

In Fig. 3, the upper lines of the shaded fields indicate the number of programmes when the coding efficiency is doubled $(\mathrm{k}=0.5)$. Multiplex can allocate three $4 \mathrm{~K}$, seven 3DTV (3D Television) or eleven HD1080p programmes, or their combination. Fig. 3 clearly shows that a reduced bit rate per programme significantly influences statistical multiplex to increase additionally the number of programmes. Despite better multiplex utilization it is still not sufficient, except perhaps for HD1080p, especially for $4 \mathrm{~K}$ formats. Looking at Fig. 1 one can see that the increase of efficiency ranges from $12 \%$, for $4 \mathrm{~K}$ UHDTV, to $26 \%$, for HD1080p programmes.

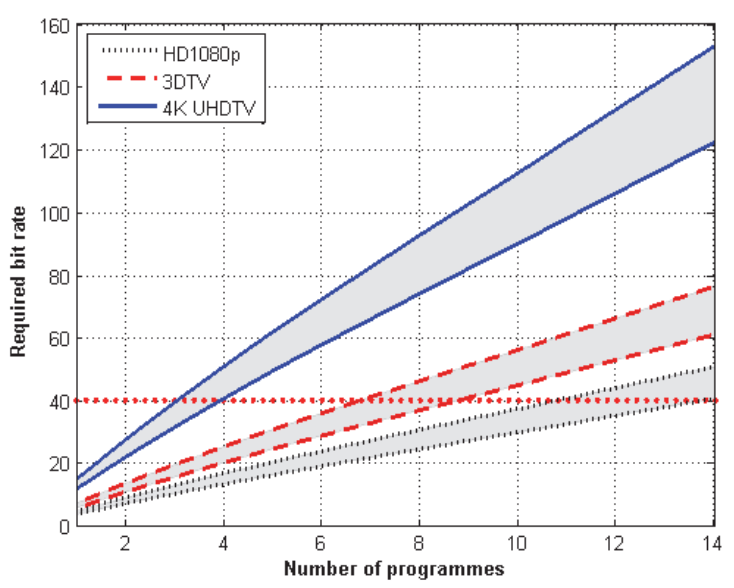

Fig. 3. Number of programmes per single channel with statistical multiplexing $(\mathrm{H} .265, \mathrm{k}=0.5$ and $\mathrm{k}=0.4)$.

Experience with coding techniques H.262 and H.264 shows that compression techniques are constantly being improved. Thus it can be expected that H.265 will also improve the compressing ratio. In Fig. 4, the shaded areas show the results in case when H.265 reduces the bitrate 
from $50 \%(\mathrm{k}=0.5)$ to $40 \%(\mathrm{k}=0.4)$ of the H.264/AVC rate assumed in this paper. The number of $4 \mathrm{~K}$ programmes per multiplex is increased from 3 to 4 . The number of 3DTV programmes increases from 7 to 9 and the number of HD1080p programmes increases from 11 to 14. Multiplex utilization is evidently better, except for the HD1080p where it is still not satisfactory.

Fig. 4. gives a good insight into the cumulative effects of compression efficiency and the application of statistical mutiplexing. Although the compression ratio increases only by $10 \%$ (from $\mathrm{k}=0.5$ to $\mathrm{k}=0.4$ ) there can be allocated up to 14 programmes in a multiplex instead of 10 or maybe 11 HD1080p programmes.

It is obvious that in the initial phase of implementing HEVC, in addition to the first generation HD formats (not shown in Fig. 4), satisfactory efficiency can be obtained by allocation of HD programmes in 1080p format. For 3DTV and 4K formats, new solutions should be explored. In addition to already improved coding techniques, for allocation of an ultra high programme rate, an effective way must be found for further increase and full utilization of this capacity.

\section{TFS MULTIPLEX}

Looking at Fig. 3, we can see that even with H.265 efficiency of $\mathrm{k}=0.4$ with $143 \mathrm{DTV}$ programmes the rate takes around $60 \mathrm{Mb} / \mathrm{s}$, while $4 \mathrm{~K}$ programmes rate takes more than $120 \mathrm{Mb} / \mathrm{s}$. Although these assumptions are approximate and compression techniques are constantly developing, it is clear that the capacity of a single RF channel is not suitable for allocation of the programme in this resolution, especially for $4 \mathrm{~K}$ or of a higher resolution.

In DVB-T2 specifications [7] and [8] although optional, the technique of combining the capacities of up to $6 \mathrm{RF}$ channels is proposed and defined in detail. This feature, called TFS (Time Frequency Slicing), implies that the segments of the programme data stream (slices), are scattered over RF channels associated with TFS group. In this case, a unique wideband multiplex covers more programmes. The obtained increase in efficiency represents a so called wideband statistical multiplexing gain.

\section{A. Wideband statistical multiplexing gain}

In Fig. 4, the blue (lower) horizontal lines show the capacity of multiplex of two and four TFS connected RF channels. HEVC with a rate reduction of $50 \%$ with respect to H.264/AVC is applied. Upper lines of the shaded segments show that in two statistically multiplexed TFS combined RF channels, 15 programmes in 3DTV format and 23 programmes in HD1080p format, can be accommodated. It guarantees a statistically balanced and efficiently utilized capacity of multiplex.

In the example of two TFS associated RF channels, it should be noted that statistical multiplexing increases the number of allocated 3DTV programmes. Although the compression rate is increased only $10 \%$ (from $\mathrm{k}=0.5$ to $\mathrm{k}$ $=0.4$ ), 19 programmes can be allocated instead of 15 . However, the number of programmes in $4 \mathrm{~K}$ format remains relatively small (it is increased from 7 to 10 ) and the capacity is still underused. But, the number of $4 \mathrm{~K}$ programmes in the four channels grows from 15 to 19 , and enters the zone of high efficiency.

\section{B. Wideband planning gain}

Besides the aggregation of capacities, TFS enables an additional increase of the multiplex capacity. RF channels can be selected from the whole available frequency band, causing different and independent transmission channel responses. The average impact on slices scattered across the combined channels, however, is much lower than the impact on single RF channels.

In DVB-T2 system, there are protection mechanisms at various levels such as LDPC and $\mathrm{BCH}$ mechanisms for FEC (Forward Error Correction), time and frequency interleaving, Gray coding, phase shift between the real and imaginary components of the signal, rotation of the constellation, etc. All these mechanisms allow more data to be transported in a single channel and reduce error to an individual bit and finally eliminate the error using CRC.

If the variability of channel response follows the variability of VBR bit stream, then TFS channel combining has the same effect on equalizing the influence of channels as statistical multiplexing has with a reduction of bit rate variability. Unfortunately, the number of RF channels assigned to terrestrial television is administratively restricted, with a tendency of gradual reduction in favor of other networks.

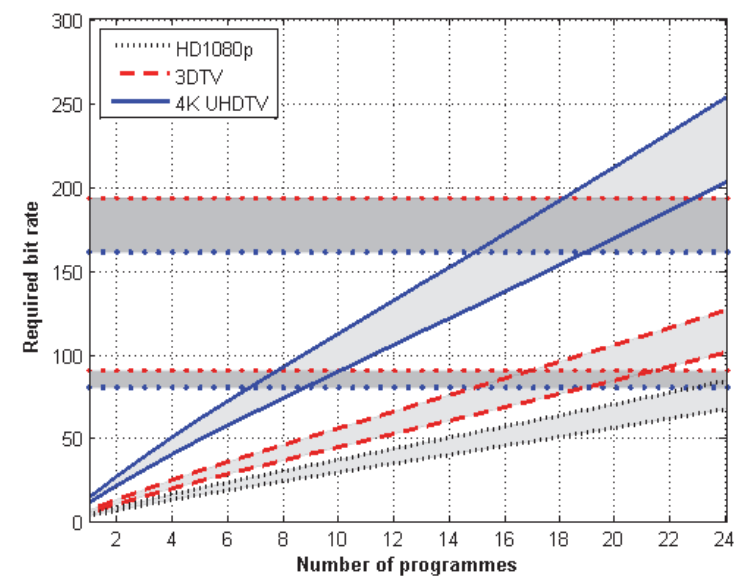

Fig. 4. TFS programmes allocation (H.265) in two and four RF channels $(\mathrm{H} .265, \mathrm{k}=0.5$ and $\mathrm{k}=0.4)$.

The measurements confirm that the gain increases with the expansion of frequency band (additional separation between RF channels) [13]. Even with a small TFS separation, the gain is significant. For instance it is $2.5 \mathrm{~dB}$ of total separation of only six RF channels. With a larger separation, the average gain is 4.5 to $5.5 \mathrm{~dB}$. Retaining other protection mechanisms unchanged, FEC correction is an easy and effective way to convert improved channel response into a reduction of protection bits and increase of the payload bit rate.

This so called planning gain can be partially or completely traded for capacity with a slightly higher code rate (FEC). Code rate, of course, should not be increased more than the planning gain affords. If the available gain 
is used only partially, the remaining gain will provide a better broadcasting quality and increase of the coverage area.

In this work, in accordance with [8], instead of the standard coding rate $2 / 3$ for a single RF channel, coding rate is assumed to be $3 / 4$, in case of TFS separated group of two channels, and $4 / 5$ for the four total separated TFS channels. The increase of capacity is shown by the upper horizontal lines for corresponding capacities in Fig. 5 .

The cumulative effect of the obtained statistical multiplexing gain and planning gain allows for each two TFS associated RF channels an increase of the number of $4 \mathrm{~K}$ programmes up to 8 in the case of $\mathrm{k}=0.5$. This is a significant improvement, although utilization capacity is still not acceptable. At the same time the number of HD1080p and 3DTV programmes is close to the zone of high capacity efficiency. However, it is shown that the number of $4 \mathrm{~K}$ programmes, multiplexed over four associated RF channels, reaches 18 (with coding rate 4/5) and it can be considered as a balanced operating mode and efficient capacity utilization.

In Fig. 5, bottom sloped lines of the shaded area show the results that can be obtained when $\mathrm{H} .265$ reduces the bit rate to $40 \%$ of that obtained with H.264/AVC. The number of $4 \mathrm{~K}$ programmes per wideband multiplex, without a planning gain over two TFS combined RF channels, can be increased to 9 , and over four channels up to 19 . This is considered as an extremely high capacity usage even in case of very demanding service formats. Including the planning gain, however, the number of $4 \mathrm{~K}$ programmes in TFS multiplex of two channels reaches an acceptable number of programmes, that is 10 . Totally 23 programmes in four channels, in the multiplex can be reached and it is the maximum capacity utilization. In this case, the synergy of the increase of capacity and statistical multiplexing is obvious, because the change from $\mathrm{k}=0.5$ to $\mathrm{k}=0.4$ expands the number of allocated programmes from 18 to 23.

\section{CONCLUSION}

This paper analyzes and quantifies the impact of the techniques such as statistical multiplexing, H.265 source video coding, and TFS channel combining, on the number of allocated HD programmes in current and upcoming formats.

HD programmes encoded by H.264/AVC within a single RF channel, even with the application of statistical multiplexing, cannot obtain sufficient capacity utilization.

H.265 improves the multiplexing of HD1080p while it is less obvious with 3DTV programmes, and the solution in case of $4 \mathrm{~K}$ resolution is still not found.
TFS multiplex over two RF channels optimizes the allocation of 3DTV programmes, but it is valid only for a few $4 \mathrm{~K}$ programmes in combination with lower definition programmes.

Only broadband statistical multiplex with four TFS combined RF channels, sufficiently separated to increase the planning gain, can be considered as a balanced operating mode for $4 \mathrm{~K}$ programmes. Effective and fully utilized broadcasting in $4 \mathrm{~K}$ format requires a reduction of H.265 compression rate to $40 \%$ of state of the art rate (H.264/AVC).

Only simultaneous application of all considered techniques gives a possibility for successful allocation of ultra high definition programmes.

\section{REFERENCES}

[1] EBU Technical Report 014: European Broadcasting Union, Status report: What follows HDTV? A status report on $1080 \mathrm{p} / 50$ and $4 \mathrm{k}$, EBU, 2012.

[2] BT.2020: International Telecommunication Union, Recommendation ITU-R BT.2020:Parameters values for ultra-high definition television systems for production and international programme exchange, ITU-R, 2012.

[3] EBU Technical Report 015: European Broadcasting Union, EBU Strategic Programme on Spectrum Management Guidelines Document: Defining Spectrum Requirements of Broadcasting in the UHF Band, EBU, 2012.

[4] "Coming Next: 3D Television over DVB-T2! “ - Teamcast, May 2010 (http:// www.teamcast.com.pdf)

[5] H.265:International Telecommunication Union, Recommendation ITU-T H.265: High efficiency video coding, ITU-T, 2013.

[6] G.J.Sullivan,J.R. Ohm, W.J.Han,T.Wiegand, "Overview of the High Efficiency Video Coding (HEVC) Standard”, IEEE Transactions on Circuits and Systems for Video Technology, Vol. 22, Dec 2012

[7] ETSI EN 302 755:European Telecommunications Standards Institute, European Standard:Digital Video Broadcasting;Frame structure channel coding and modulation for a second generation digital terresttrial television broadcasting system (DVB-T2), ETSI, 2009.

[8] ETSI A 133: “Digital Video Broadcasting (DVB); Implementation guidelines for a second generation digital terrestrial television broadcasting system (DVB-T2) “, Feb 2012.

[9] M. Rezaei, I. Bouazizi, M. Gabbouj, "Statistical Time-Frequency Multiplexing of HD Video Traffic in DVB-T2", Hindawi publishing Corporation, International Journal of Digital Multimedia Broadcasting, vol.2009, 2008.

[10] McCAN, K, Adriana Mattei, „Technical Evolution of the DTT Platform", Independent Report from ZetaCast Limited, commissioned by Ofcom, Jan 2012.

[11] J.R.Ohm, G.J.Sullivan, H. Schwarz, T.K. Tan, T.Wiegand, "Comparsion of the Coding Efficiency of Video Coding StandardsIncluding High Efficiency Video Coding (HEVC)", IEEE Transactions on Circuits and Systems for Video Technology, 2012.

[12] P. Hanhart, M. Rerabek, F. Simone, T. Ebrahimi,'Subjective quality evaluation of the upcoming HEVC video compression standard", Proceedings of SPIE Optical Engineering and Applications, vol.8499, San Diego, Aug. 2012.

[13] EBU-TECH 3348:European Broadcasting Union, Status Report: Frequency and Network Planning Aspects of DVB-T2, EBU, 2012. 